|| Print ISSN: 2589-7837 || Online ISSN: 2581-3935 ||

International Journal of Medical Science and Diagnosis Research (IJMSDR)

Available Online at www.ijmsdr.com

NLM (National Library of Medicine ID: 101738824)

Original Research Article

Volume 5, Issue 9; September:2021; Page No. 42-47

\title{
Disease Modifying Treatment of Osteoarthritis with Triple Combination Anti-Microbial Therapy
}

\author{
Ernest L. Bonner, Jr., M.D., J.D. \\ Medical Doctors Group INC
}

\section{Conflicts of Interest: Nil}

Corresponding author: Ernest L. Bonner

DOI: https://doi.org/10.32553/ijmsdr.v5i9.851

\section{Abstract:}

Background: The study author, while still a medical student, observed microscopic evidence of chronic inflammation in joint tissue of patients suffering with osteoarthritis and hypothesized that osteoarthritis was caused by an infectious process. After graduating from medical school and entering into private practice, the study author had several patients who informed him that when they were on tetracycline therapy, for reasons other than arthritis, their arthritis symptoms diminished to some extent. After reporting improvement of arthritic symptoms with tetracycline, one of these patients subsequently received metronidazole and while taking both tetracycline and metronidazole reported that his arthritic symptoms improved to an even greater extent, as his joint pain further diminished but did not resolve. Subsequent treatment of osteoarthritis in other patients included the combination of tetracycline, metronidazole, other antibiotics and some natural products but only when L-lysine was added to the tetracycline and metronidazole combination therapy did the author observe yet further improvement in arthritic pain. Since L-lysine was reported to inhibit the growth of herpes simplex virus, the author and another colleague concluded that acyclovir might be a beneficial addition to the tetracycline, metronidazole, L-lysine combination. This four-component combination treatment resulted in total resolution or a marked diminution of osteoarthritis joint pain, joint tenderness and joint stiffness in most patients treated. It was further determined that L-lysine could be eliminated; that acyclovir could be replaced with valicyclovir; and that tetracycline could be replaced with minocycline or doxycycline.

Preliminary studies of patients diagnosed with osteoarthritis and treated with tetracycline alone or the combination of tetracycline and metronidazole demonstrated improvement in their osteoarthritic symptoms. However the triple combination of doxycycline (broad spectrum antibiotic), metronidazole (antiprotozoal and antibacterial imidazole) and acyclovir (antiviral purine nucleoside) administered orally twice daily for eight weeks showed marked success in a group of patients suffering with osteoarthritis by substantially eliminating their joint pain, joint tenderness and joint stiffness while overcoming their fatigue and increasing their energy level. The data in this report substantiate that this triple combination is a disease modifying therapy that eliminates or markedly diminishes the symptomatology of osteoarthritis and further supports the hypothesis that osteoarthritis is an infectious disease. Objective: To evaluate the effect of the triple combination therapy of doxycycline, metronidazole, and acyclovir on the joint pain, joint stiffness and functionality in patients suffering with osteoarthritis. Design: Non-blinded study of treatment of patients suffering with osteoarthritis Setting: Primary care internal medicine practice in Alameda County, California. Sample: Six adult individuals suffering with osteoarthritis. Patients: Six patients, ages 53 through 82 years. Patient 1 was treated for 28 days and Patients 2 through 6 were treated for 56 days. Method: After obtaining informed consent, six patients suffering with osteoarthritis were prescribed a 56 day course of treatment with the triple combination of doxycycline, metronidazole, and 
acyclovir for treatment of their osteoarthritis. Utilizing the WOMAC index for assessment of the effectiveness of treatment of osteoarthritis, pre-treatment pain, stiffness and functional limitation, were compared to post-treatment pain, stiffness and functional limitations. Results: Three patients demonstrated total resolution of pain and stiffness and greater than $95 \%$ improvement in functional limitations. One of the remaining three patients demonstrated a 70\% improvement in pain, total resolution of stiffness, and an $85 \%$ improvement in functional limitations; another one of the three remaining patients demonstrated an $86 \%$ improvement in pain, total resolution of stiffness, and a $79 \%$ improvement in functional limitations; while the last of the three remaining patients demonstrated at least an $85 \%$ improvement in pain, $67 \%$ improvement in stiffness, and a $63 \%$ improvement in functional limitations. Limitations: No randomization. No placebo. No control group. Small sample size. Conclusion: The results of treatment of these patients suffering the osteoarthritis support the hypothesis that osteoarthritis is an infectious disease. These results further suggest that osteoarthritis is, at a minimum, modifiable with the triple combination therapy of doxycycline, metronidazole, and acyclovir and is even curative of with this breakthrough treatment in many cases. The medical treatment reported in this article represents the first truly Disease Modifying medication therapy developed for osteoarthritis.

Keywords: WOMAC index

\section{Introduction:}

\section{Osteoarthritis Study with Anti-infectious Medications}

Osteoarthritis affects hundreds of millions of people globally and is the most common of all the arthritic diseases, accounting for roughly half of those individuals who suffer with

arthritis. Almost all individuals suffering from osteoarthritis experience some degree of movement limitation. This disease is a leading cause of disability and functional limitations of adults. Data from 1997 confirms that osteoarthritis in the U.S. accounted for approximately 55 percent of all arthritis related hospitalizations (1) and about 7-8 million arthritis related ambulatory medical care visits (2). During that same year in the U.S., knee and hip replacement surgery resulting from osteoarthritis cost around $\$ 8$ billion $(\mathbf{1 , 3 )}$. Thus osteoarthritis is truly a great cost burden to a healthcare system and to affected individuals.

Pharmacological treatments for osteoarthritis have been largely limited to symptom management only. Glucosamine sulfate and chondroitin sulfate, however, are dietary supplements which have demonstrated evidence for at least a transient minor disease-modifying effect on osteoarthritis (4). This present report identifies a new breakthrough treatment which attacks the disease at its core and thereby eliminates the accompanying symptoms.

Osteoarthritis has been commonly believed to be related to aging and/or joint cartilage wear and tear $(\mathbf{5 , 6 )}$. This study, however, reports a newly developed treatment, which strongly supports the hypothesis that Osteoarthritis is actually caused by an infectious process. In contrast to traditional treatment limited to addressing the symptom of only osteoarthritis pain, this newly developed treatment attacks the disease and is even curative in many cases. This patented treatment consists of doxycycline, metronidazole, and acyclovir. In this present study, knee osteoarthritis is evaluated using the Western Ontario and McMaster Universities (WOMAC) Index (7).

Significant improvement was acknowledged by all the participants. Each participant in this study was diagnosed with osteoarthritis of the knee. Each participant was prescribed a 56 day course of treatment with the triple therapy combination (doxycycline, metronidazole, and acyclovir) and in each case pain, stiffness, and functional limitations improved significantly. The mean improvement in the Pain, Stiffness and Functional Limitations score was 90.1 percent, 94.5 percent, and 86.5 percent respectively. The results of this study confirm the triple therapy combination (doxycycline, metronidazole, and 
acyclovir) represents a major advancement in the treatment of osteoarthritis.

\section{Method}

Six patients, ages 53 through 82 years, participated in this study. Each of these selected participants suffered from osteoarthritis and completed a WOMAC Osteoarthritis Index form for osteoarthritis of the knee prior to initiation of treatment. Each of the participants also completed a WOMAC Osteoarthritis Index form following completion of treatment. The triple combination therapy of doxycycline, metronidazole, and acyclovir was used to treat each of the six patients. One patient completed only 28 days of such treatment and was thereafter lost to follow-up while the other five patients completed 56 days of treatment. The primary outcomes measured in this study included joint pain, stiffness, and functional limitations associated with moderate to severe osteoarthritis.

\section{Protocol}

Six patients were selected for the eight week course of treatment with doxycycline $\mathrm{HCl}$, metronidazole and acyclovir dosed orally twice daily.

1. Each patient satisfied the American College of Rheumatology diagnostic criteria for osteoarthritis as defined by its criteria for 'Classification of Idiopathic Osteoarthritis (OA) of the Knee [Appendix A].

2. All six patients presented with complaints of multiple joint pains including knee pain.
3. The six patients ranged in age from 53 to 82 years.

4. Each of the six patients completed a WOMAC Osteoarthritis Index form for osteoarthritis of the knee prior to initiation of treatment with the triple combination therapy.

5. Each of the six patients completed the WOMAC Osteoarthritis Index form for osteoarthritis of the knee following completion of treatment with the triple combination therapy.

6. One of the six patients was treated with the triple combination therapy for 28 days and five of the six patients were treated for 56 days.

7. The triple combination therapy consisted of a combination of doxycycline $100 \mathrm{mg}$, metronidazole $250 \mathrm{mg}$, and acyclovir $400 \mathrm{mg}$ taken orally twice daily.

\section{Results}

Pain, stiffness, and functional limitations resulting from osteoarthritis were assessed in six patients treated with the combination triple therapy of doxycycline, metronidazole, and acyclovir. These parameters were assessed by comparing the pretreatment and post treatment WOMAC Osteoarthritis Index patient score for pain, stiffness, and functional limitations in each of the participants. The efficacy data is presented subject-by-subject in a series of tables (Tables 1-4) in which pain, stiffness, and functional limitations are quantified according to defined scales of measurement pertaining to the WOMAC Osteoarthritis Index. In addition, an average of the patients scores is included at the bottom.

Table 1: Pain Comparison

\begin{tabular}{|l|l|l|l|}
\hline Patients & $\begin{array}{l}\text { Pre-treatment } \\
\text { Pain Score }\end{array}$ & $\begin{array}{l}\text { Post-treatment Pain } \\
\text { Score }\end{array}$ & Improvement [\%] \\
\hline 1 & 12 & 0 & 100 \\
\hline 2 & 14 & 0 & 100 \\
\hline 3 & 14 & 0 & 100 \\
\hline 4 & 10 & 3 & 70 \\
\hline 5 & 14 & 2 & 86 \\
\hline 6 & 13 & 2 & 85 \\
\hline Average & $\mathbf{1 2 . 8}$ & $\mathbf{1 . 2}$ & $\mathbf{9 0 . 1}$ \\
\hline
\end{tabular}


Table 2: Stiffness Comparison

\begin{tabular}{|l|l|l|l|}
\hline Patients & $\begin{array}{l}\text { Pre-treatment } \\
\text { Stiffness Score }\end{array}$ & $\begin{array}{l}\text { Post-treatment } \\
\text { Stiffness Score }\end{array}$ & Improvement [\%] \\
\hline 1 & 4 & 0 & 100 \\
\hline 2 & 5 & 0 & 100 \\
\hline 3 & 6 & 0 & 100 \\
\hline 4 & 5 & 0 & 100 \\
\hline 5 & 6 & 0 & 100 \\
\hline 6 & 6 & 2 & 67 \\
\hline Average & $\mathbf{5 . 3}$ & $\mathbf{0 . 3 3}$ & $\mathbf{9 4 . 5}$ \\
\hline
\end{tabular}

Table 3: Functional Comparison

\begin{tabular}{|l|l|l|l|}
\hline Patients & $\begin{array}{l}\text { Pre-treatment } \\
\text { Functional Limitation } \\
\text { Score }\end{array}$ & $\begin{array}{l}\text { Post-treatment } \\
\text { Functional Limitation } \\
\text { Score }\end{array}$ & Improvement [\%] \\
\hline 1 & 36 & 0 & 100 \\
\hline 2 & 48 & 2 & 96 \\
\hline 3 & 52 & 2 & 96 \\
\hline 4 & 40 & 6 & 85 \\
\hline 5 & 39 & 8 & 79 \\
\hline 6 & 35 & 13 & 63 \\
\hline Average & $\mathbf{4 1 . 7}$ & $\mathbf{5 . 1}$ & $\mathbf{8 6 . 5}$ \\
\hline
\end{tabular}

Table 4: Overall Efficacy

\begin{tabular}{|l|l|l|l|}
\hline Patients & $\begin{array}{l}\text { Global Pre-treatment } \\
\text { Score } \\
\text { (Pain+Stiffness+Functional } \\
\text { Limitation) }\end{array}$ & $\begin{array}{l}\text { Global Post-treatment } \\
\text { Score } \\
\text { (Pain+Stiffness+Functional } \\
\text { Limitation) }\end{array}$ & $\begin{array}{l}\text { Global } \\
\text { Improvement } \\
{[\%]}\end{array}$ \\
\hline 1 & 52 & 0 & 100 \\
\hline 2 & 67 & 2 & 97 \\
\hline 3 & 72 & 2 & 97 \\
\hline 4 & 55 & 9 & 84 \\
\hline 5 & 59 & 10 & 83 \\
\hline 6 & 54 & 17 & 69 \\
\hline Average & $\mathbf{5 9 . 8}$ & $\mathbf{6 . 7}$ & $\mathbf{8 8 . 3}$ \\
\hline
\end{tabular}

Pain decreased significantly with the triple combination therapy in all six patients and completely resolved in three of the six patients. Pain was greatly reduced by such treatment in the remaining three patients. One of those three patients who continued to experience pain after completion of treatment noted a $70 \%$ reduction in pain score while the other two patients experienced an $86 \%$ and $85 \%$ reduction in pain score respectively. The mean improvement in pain for all six patients was $\mathbf{9 0 . 1 \%}$.

Stiffness also decreased significantly in all 6 (six) patients who received the triple combination therapy. Resolution of stiffness occurred in 5 (five) of those 6 (six) patients who received the triple combination therapy. One of the six patients continued to experience stiffness after completion 
of treatment but noted a $67 \%$ improvement in Stiffness score. The mean improvement in the Stiffness score was $94.5 \%$.

Functional limitations additionally improved significantly in each of the 6 (six) patients who received the triple combination therapy. One of the six patients experienced total resolution of functional limitations. Two of the six patients noted a $96 \%$ improvement in their functional limitations score; one patient noted an $85 \%$ improvement in functional limitations score; one patient noted a $79 \%$ improvement in functional limitations score; and one patient noted a $63 \%$ improvement in functional limitations score. The mean improvement in the functional limitations score was $86.5 \%$.

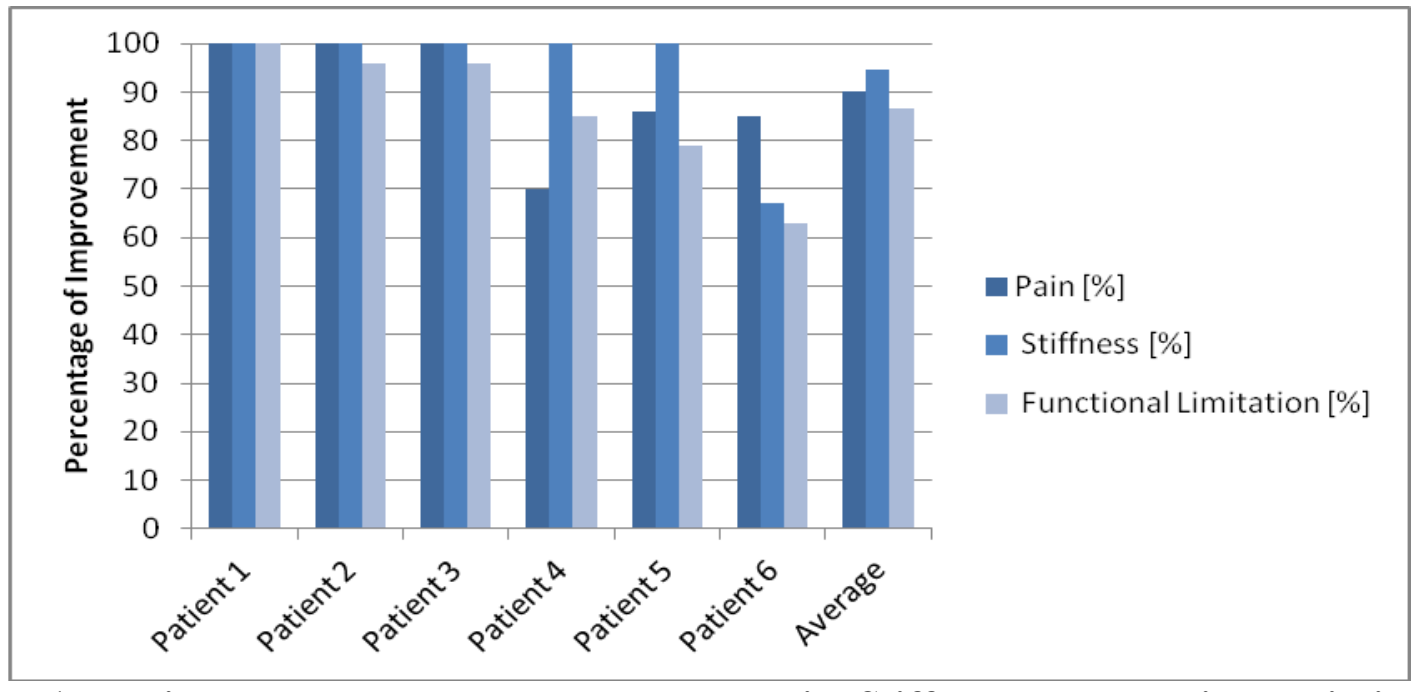

Figure 1: Patient Improvement Percentage on Pain, Stiffness and Functional Limitation.

The overall efficacy of treatment was assessed by global scores. These global scores demonstrated significant improvement in each of the six patients treated with the triple combination therapy. One of the six patients demonstrated total resolution of pain, stiffness, and functional limitations or 100\% improvement in that patient's global score. Two of the six patients achieved a $97 \%$ improvement in their global score; one patient demonstrated an $84 \%$ improvement in the global score; one patient demonstrated an $83 \%$ improvement in the global score; and one patient demonstrated a $69 \%$ improvement in the global score.

\section{Discussion}

Osteoarthritis has typically been stated to be a consequence of aging and/or joint cartilage wear and tear. Until recently osteoarthritis was considered a non-inflammatory arthritic disease but in recent times the presence of an inflammatory process has been generally acknowledged (8). The etiology of this inflammatory process, however, has never been elucidated.

Most treatments of osteoarthritis target only the symptoms of the disease. The triple combination antimicrobial treatment announced in this report, however, targets the disease itself, thereby eliminating the accompanying disease symptomsincluding the symptoms of pain and stiffness. The results of this study, and our prior study, supports the hypothesis that osteoarthritis is an infectious disease which can be effectively treated with this triple combination antimicrobial therapy announced in this report and that prior report.

In this present study six patients were treated with anti-infectious medications and each patient achieved a major benefit from such treatment. If the patient with the known ligament tear is excluded from the analysis, the mean improvement in pain increases from 90.17 to 91.2 , in stiffness 
from 94.5 to 100 and in functional limitations from 86.5 to 91.2. This anti-infectious medication treatment consisted of doxycycline, metronidazole, and acyclovir. Medication treatment of osteoarthritis, heretofore, has been largely limited to treatment of symptoms only, with pain being the primary symptom target. The treatment announced in this present study suggests osteoarthritic disease modification in each patient in the study with cure of patients 1,2 , and 3 . Again the treatment more importantly strongly implies and supports the hypothesis that osteoarthritis is an infectious disease. Though the causative organism has not yet been identified, an effective treatment of the disease has been developed and is documented in this present study as well as our prior study.

Other studies utilizing Glucosamine And/or Chondroitin sulfate And/or celecoxib have documented at best a $20 \%$ improvement in WOMAC Scores for pain (9). Whereas the WOMAC scores on pain from baseline for this study showed an average of $90 \%$ improvement in 8 weeks [95\% confidence level $3.43-8.570$ due to the small sample size] for this Breakthrough Treatment.

\section{Conclusion}

Osteoarthritis is likely an infectious disease which will respond to a combination triple combination antimicrobial treatment. This triple combination anti-infection treatment consists of doxycycline, metronidazole, and acyclovir. This treatment is the first truly disease modifying medication treatment that has been developed for osteoarthritis and in many cases this treatment is curative.

\section{References}

1. Lethbridge-Cejku M, Helmick CG, Popovic JR. Hospitalizations for arthritis and other rheumatic conditions: Data from the 1976 National Hospital Discharge Survey. Medi Care 2003;41(12):1367-1373.
2. Arthritis Care Res 2010;62(4):460-4. [Data Source: 2001-2005 National Ambulatory Medical Care Survey and 2001-2005 National Hospital Ambulatory Medical Care Survey]

3. Centers for Disease Control and Prevention. Reviewed: $2009 . \quad$ Updated: 2011. Osteoarthritis. Retrieved from http://www.cdc.gov/arthritis/basics/osteoarthri tis.htm

4. Sawitzke, A. D. Shi, H., Finco, M. F., Dunlop, D. D., Harris, C. L., Singer, N. G., Bradley, J. D., Silver, D., Jackson, C. G., Lane, N. E., Oddis, C. V., Wolfe, F., Lisse, J., Furst, D. E., Bingham III, C. O., Reda, D. J., Moskowitz, R. W., Williams, H. J., Clegg, D. O. (2010). Clinical efficacy and safety over two years use of glucosamine, chondroitin sulfate, their combination, celecoxib or placebo taken to treat osteoarthritis of the knee: a GAIT report. Ann Rheum Dis. 2010 Aug;69(8):1459-1464.

5. Teitel, A. D. (updater). (2011). Osteoarthritis. MedlinePlus: Trusted Health Information for You. Retrieved from http:/www.nlm.nih.gov/medlineplus/ency/arti cle/000423.htm

6. Teitel, A. D., Zieve, D. (Reviewers). (2011). Osteoarthritis. PubMed Health. Retrieved from

http://www.ncbi.nlm.nih.gov/pubmedhealth/P MH0001460/

7. Bellamy N, Buchanan WW, Goldsmith $\mathrm{CH}$, Campbell J, Stitt LW. (1988). Validation study of WOMAC: a health status instrument for measuring clinically important patient relevant outcomes to antirheumatic drug therapy in patients with osteoarthritis of the hip or knee. J Rheumatol 1988;15:1833-40.

8. Berenbaum, F. Osteoarthritis as an inflammatory disease (osteoarthritis is not osteoarthrosis!) (2013) Osteoarthritis and Cartilage, 21 (1), pp. 16-21. doi: 10.1016/ j.joca.2012.11.012 